\title{
Spontaneous Pneumomediastinum in COVID-19 Pneumonia: A Rare Occurence
}

\author{
Saerah Iffat Zafar ${ }^{1}$, Hina Nasir ${ }^{1}$, Aliya Halim ${ }^{1}$, Atiq Ur Rehman Slehria ${ }^{1}$ and Nadeem Zafar ${ }^{2}$ \\ ${ }^{1}$ Armed Forces Institute of Radiology and Imaging (AFIRI), Rawalpindi, Pakistan \\ ${ }^{2}$ Armed Forces Institute of Pathology (AFIP), Rawalpindi, Pakistan
}

\begin{abstract}
Spontaneous pneumomediastinum with COVID pneumonia is a rare occurrence with or without accompanying subcutaneous emphysema or pneumothorax. The aim of this study was to establish relation of this complication to severity of lung disease and its clinical outcome. The study was conducted for a period of seven months from April to October 2020 in the CT Department of Armed Forces Institute of Radiology and Imaging (AFIRI), Rawalpindi, Pakistan. All COVID positive patients having spontaneous pneumomediastinum on high resolution CT (HRCT) chest were included $(n=14)$. These patients were assessed for severity of lung disease as per CT severity score (CTSS), and were followed up for their clinical outcome. All patients with spontaneous pneumomediastinum had moderate to severe degree of COVID pneumonia; mortality in patients with pneumomediastinum was $50 \%$; and was seen in those patients who had greater severity of lung disease as per the CTSS.
\end{abstract}

Key Words: Spontaneous, Pneumomediastinum, COVID, Pneumothorax, Subcutaneous, Emphysema.

How to cite this article: Zafar SI, Nasir H, Halim A, Slehria AUR, Zafar N. Spontaneous Pneumomediastinum in COVID-19 Pneumonia: A Rare Occurence. J Coll Physicians Surg Pak 2022; 32(02):230-232.

\section{INTRODUCTION}

SARS-Cov-19 has changed the world ever since it was declared a pandemic in March 2020. Radiology plays a vital role in detection, management, and surveillance of COVID patients in determining the effects of the virus on lungs; and is helpful in prediction of clinical outcome. CT severity score is being used worldwide, according to the hospital protocol, which correlates well with the prognosis and ultimate outcome of these patients. ${ }^{1}$ Pneumomediastinum implies presence of air in the mediastinum; if it occurs without any history of trauma or intervention, then it is labelled as spontaneous. Secondary pneumomediastinum may occur in association with certain conditions such as asthma, tracheomalacia, in patients having steroids or on other medicines, or in respiratory infections. ${ }^{2}$ Pneumomediastinum is a rare complication of COVID-19 pneumonia. It can be seen in milder forms of COVID pneumonia with spontaneous resolution on follow-up. ${ }^{3}$ Alternatively, in patients having severe pneumonia, pneumomediastinum is associated with significant morbidity and mortality.

Correspondence to: Dr. Saerah Iffat Zafar, Armed Forces Institute of Radiology and Imaging (AFIRI), Rawalpindi, Pakistan

E-mail: saerah_syk07@yahoo.com

Received: February 01, 2021; Revised: November 01, 2021;

Accepted: November 19, 2021

DOI: https://doi.org/10.29271/jcpsp.2022.02.230
In COVID patients, pneumomediastinum can be spontaneous or due to barotrauma in patients, who are intubated. A study in New York Hospital determined the rate of barotrauma in COVID patients as $24 \%$ compared to intubated non-COVID patients (11\%) due to the degree of alveolar damage, induced by the virus. ${ }^{4}$ In COVID pneumonia, presence of free air in mediastinum can be a harbinger of poor clinical outcome, if seen in association with increasing severity of underlying lung disease.

The aim of this study was to assess the COVID pneumonia patients for development of spontaneous pneumomediastinum and to determine its relation to severity of pneumonia and the clinical outcome of disease.

\section{METHODOLOGY}

Out of known COVID-19 disease patients (1979 patients) referred to the CT department of AFIRI, Rawal pindi for HRCT over a period of seven months (April- October 2020) - 14 had pneumomediastinum on imaging $(0.7 \%)$. For these patients, age, gender, type of lung findings - ground glass opacities (GGOs)/consolidation/interlobular septal thickening, or mixed pattern- were recorded. Patients having pneumomediastinum secondary to intubation were notincluded inthestudy.

Pneumomediastinum was divided into mild, moderate and severe categories, depending upon the distribution of air within mediastinum and associated findings. Division of these categories was as follows: mild, if minimal mediastinal air from larynx to carina level plus restricted cervical subcutaneous emphysema; moderate in the presence of mild criteria plus involvement of peribronchial tree and pericardial area with 
subcutaneous emphysema; and severe in the presence of moderate criteria plus extensive chest wall subcutaneous emphysema. ${ }^{5}$

Pneumonia was categorised into varying degrees of severity, according to CT severity score (CTSS), for the purpose of which, both lungs were divided into 10 segments, considering apico-posterior and antero-medial basal in left lung as separate segments. One point was assigned to each segment (10 on either side), if there was $<50 \%$ segment involvement, or two points if it was $>50 \%$ (maximum 20 points for either lung). Classification of CTSS was assigned as minimal changes (score $=1-10)$, mild $($ score $=11-20)$, moderate $($ score $=21-30)$, and severe $($ score $=$ 31-40).

\section{RESULTS}

The age of patients having pneumomediastinum with COVID 19 ranged from 11 to 82 years with mean age of 59 years. Out of 14 patients, only one was female. Over all patients with COVID-19 also showed male preponderance ( $22 \%$ versus $78 \%$, female to male ratio). The predominant pattern of lung involvement included mixed pattern - consolidations along with ground glass opacities (GGOs) in 13 out of 14 patients; while one patient demonstrated interlobular septal thickening as predominant pattern. In patients showing pneumomediastinum on HRCT chest of Rt PCR positive COVID-19 patients, CTSS ranged from 23-40; five patients had moderate disease (35.7\%), while nine had severe disease (64.2\%). No pneumomediastinum was noted in patients having minimal to mild CTSS. Figure 1 shows the relation between degree of pneumomediastinum and lung disease severity of COVID 19 as determined by CTSS. In most of the patients, severity of pneumomediastinum increased with severity of lung disease. Ten patients had associated subcutaneous emphysema; while pneumothorax was present in eight patients. Out of these eight patients, two had tension pneumothorax; while rest had minimal pneumothorax. Mortality was $50 \%$; patients having moderate to severe degree of pneumomediastinum had a poorer clinical outcome than those with mild pneumomediastinum.

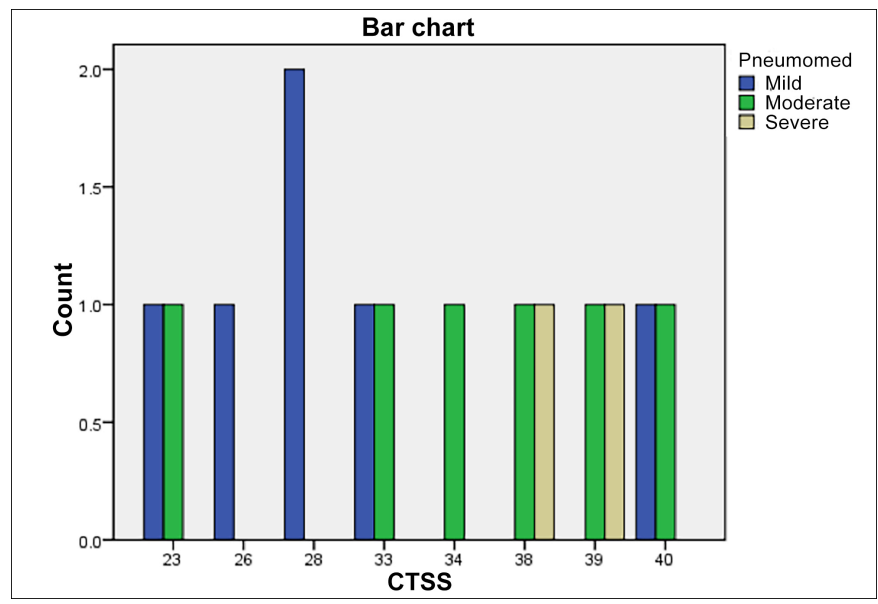

Figure 1: Graph relating degree of pneumomediastinum to CT severity score (CTSS) - mild degree predominantly with moderate disease, while moderate to severe pneumomediastinum seen with increasing lung disease severity.

\section{DISCUSSION}

Pneumomediastinum occurs when there is pressure gradient between the alveoli and interstitium, leading to rupture of alveoli. ${ }^{6}$ It can be spontaneous or secondary. Spontaneous pneumomediastinum is predominantly seen in male patients; ${ }^{7}$ as was the case in the patients of this study ( 13 out of 14 patients were males). This complication is a rare occurrence in patients with COVID-19 pneumonia. Initially it was reported as individual cases from Italy and China ${ }^{8}$ - both patients survived the acute episode of illness. It was also reported secondarily in ventilated patients as barotrauma proves a higher risk for COVID-19, due to excessive alveolar damage and tracheobroncheolar injury compared to non-COVID patients. ${ }^{9}$ Intubated patients were however excluded from our study.

Increase in lung disease severity on HRCT is indicated by the formation of consolidations in areas of ground glass opacities, which appears commonly at the middle-late stage (up to two weeks) of disease - representing progressive process. ${ }^{10}$ Thirteen of the patients of pneumomediastinum had widespread GGOs with basal consolidations (indicative of intermediate to late disease). None of the patients had GGOs alone, which is an early finding of COVID pneumonia when seen in isolation; ${ }^{11}$ while one patient had interlobular septal thickening with GGOs. In non-COVID patients, spontaneous pneumomediastinum generally occurs in a younger age group - 20-40 years with mean age of 25 years in one study; ${ }^{12}$ while in this study with COVID pneumonia, majority of patients ( $71 \%$ - $n=10 / 14)$ were at or above 60 years with mean age of presentation at 59 years.

One patient had tension pneumomediastinum, causing compression of the mediastinal vessels, who could not survive. Extensive pneumomediastinum with tension compresses upon the major neck vessels, resulting in poor prognosis. Minimal pneumothorax was not found to be a potential predictor of poor outcome in patients of this study; and was seen in $57 \%$ of patients $(n=8)$. It affected the clinical outcome only if it was large or associated with tension. Pneumomediastinum was unaccompanied by pneumopericardium in our study, while mortality of patients with pneumomediastinum was $50 \%$.

\section{CONCLUSION}

Spontaneous pneumomediastinum is rare in COVID pneumonia; and is seen at the intermediate to late stages of the disease process. Severity of underlying pneumonia remains the most important predictor of prognosis in patients having spontaneous pneumomediastinum.

\section{ETHICALAPPROVAL:}

Approval was obtained prior to commencement of this study by the Ethical Review Committee of Armed Forces Institute of Radiology and Imaging (AFIRI), Rawalpindi.

\section{PATIENTS' CONSENT:}

Patients' consent was not required.

\section{CONFLICT OF INTEREST:}

The authors declared no conflict of interest. 


\section{AUTHORS' CONTRIBUTION:}

SIZ: Concept, data collection, review of findings, result compilation and statistical evaluation; review of literature.

$\mathrm{HN}$ : Assessment of variables, recording of findings and collection of data, compilation of results.

$\mathrm{AH}$ : Assessment of variables, collection of data, recording of data

ARS: Review of literature.

NZ: Rt PCR evaluation of COVID/suspected COVID patients.

\section{REFERENCES}

1. Yang R, Li X, Liu H, Zhen Y, Zhang X, Xiong Q, et al. Chest CT severity score: An imaging tool for assessing severe Covid 19. Radiol Cardiothorac Imaging 2020; 2(2): e200047. doi: 10.1148/ryct.2020200047.

2. Zylak CM, Standen JR, Barnes GR, Zylak CJ. Pneumomediastinum revisited. Radiographics 2000; 20(4): 1043-57. doi: 10.1148/radiographics.20.4.g00jl131043

3. Brogna B, Bignardi E, Salvatore $P$, Alberigo M, Brogna C, Megliola $A$, et al. Unusual presentations of COVID-19 pneumonia on CT scans with spontaneous pneumomediastinum and loculated pneumothorax: A report of two cases and a review of the literature. Heart Lung 2020; 49(6):864-8. doi: 10.1016/j.hrtlng.2020.06.005.

4. Palmer WJ. X-ray identifies more air pressure lung injuries in COVID-19 patients. diagnostic imaging, July 72020 available from http://www.diagnosticimaging.com/view/ xray-identifies-more-air-pressure-lung-injuries-in-covid-19.

5. Kim KS, Jeon HW, Moon Y, Du Kim Y, Im Ahn M, Kil Park, et al. Clinical experience of spontaneous pneumomediastinum: Diagnosis and treatment. J Thorac Dis 2015;
7(10):1817-24. doi:10.3978/j.issn.2072-1439.2015.10.58.

6. Kolani S, Houari N, Haloua M, Lamrani YA, Boubbou M, Serraj $M$, et al. Spontaneous pneumomediastinum occurring in patients with SARS-COV-2 patients. ID Cases 2020; 21 e00806. doi: 10.1016/j.idcr. 2020.e00806.

7. Dionísio P, Martins L, Moreira S, Manique A, Macedo R, Caeiro F, et al. Spontaneous pneumomediastinum: Experience in 18 patients during the last 12 years. J Bras Pneumol 2017; 43(2):101-5. doi:10.1590/S180637562016000000052.

8. Zhou C, Gao C, Xie Y, Xu M. COVID-19 with spontaneous pneumomediastinum. Lancet Infectious Dis 2020; 20(4):510. doi: 10.1016/s1473-3099(20)30156-0.

9. Wali A, Rizzo V, Bille A, Routledge T, Chambers AJ. Pneumomediastinum following intubation in COVID 19 patients: A case series. Anaesthesia. 2020; 75(8):1076-81. doi.org/10.1111/anae.15113.

10. Carotti M, Salaffi F, Sarzi-Puttini P, Agostini A, Borgheresi $A$, Minorati $D$, et al. Chest CT features of coronavirus disease 2019 (COVID-19) pneumonia: Key points for radiologists. Radiol Med 2020; 125(7):636-46. doi:10. 1007/s11547-020-01237-4.

11. Zhan J, Li H, Yu H, Liu X, Zeng X, Peng D, et al. 2019 novel coronavirus (COVID-19) pneumonia: CT manifestations and pattern of evolution in 110 patients in Jiangxi, China. Eur Radiol 2021; 31(2):1059-1068. doi.org/10.1007/ s00330-020-07201-0.

12. Dirweesh A, Alvarez C, Khan M, Christmas D. Spontaneous pneumomediastinum in a healthy young female: A case report and literature review. Respir Med Case Rep 2017; 20:129-32. doi:10.1016/j.rmcr.2017.01.014. 